$\begin{array}{ll}\text { Abstracta Iranica } & \begin{array}{l}\text { Abstracta Iranica } \\ \text { Revue bibliographique pour le domaine irano-aryen }\end{array} \\ & \text { Volume } \mathbf{2 3} \mid \mathbf{2 0 0 2} \\ & \text { Comptes rendus des publications de } \mathbf{2 0 0 0}\end{array}$

\title{
Excavation at Ghubayrā, Iran. London, SOAS, University of London, 2000, 508 p. dont 87 fig. et
} $144 \mathrm{pl}$.

\section{Monik Kervran}

\section{(2) OpenEdition Journals}

Édition électronique

URL : http://journals.openedition.org/abstractairanica/35457

DOI : 10.4000/abstractairanica.35457

ISSN : 1961-960X

Éditeur :

CNRS (UMR 7528 Mondes iraniens et indiens), Éditions de l'IFRI

\section{Édition imprimée}

Date de publication : 15 mai 2002

ISSN : 0240-8910

Référence électronique

Monik Kervran, « Excavation at Ghubayrā, Iran. London, SOAS, University of London, 2000, 508 p. dont 87 fig. et 144 pl. », Abstracta Iranica [En ligne], Volume 23 | 2002, document 183, mis en ligne le 08 février 2010, consulté le 25 septembre 2020. URL : http://journals.openedition.org/abstractairanica/ 35457 ; DOI : https://doi.org/10.4000/abstractairanica.35457

Ce document a été généré automatiquement le 25 septembre 2020

Tous droits réservés 


\title{
Excavation at Ghubayrā, Iran. London, SOAS, University of London, 2000, 508 p. dont 87 fig. et $144 \mathrm{pl}$.
}

\author{
Monik Kervran
}

1 Cet ouvrage dont le but essentiel est de présenter les résultats d'une fouille menée entre 1971 et 1976 sur le site de Ghubayrā, dans la province de Kirmān, fournit aussi des informations sur les capitales régionales qui se sont succédé de l'antiquité à l'époque moderne. Aux temps d'Alexandre jusqu'au début de la période sassanide, la capitale provinciale fut Jïruft, certainement moins chaude, mieux irriguée et boisée à cette époque qu'aujourd'hui, comme en témoignent les lits asséchés de rivières et les restes polliniques. Dès l'époque sassanide le changement climatique se fit sentir et, d'après Idrīsī, sous Hormizd I (deuxième moitié du $3^{\mathrm{e}} \mathrm{s}$.), l'administration provinciale quitta Jīruft pour le village d'altitude de Qaryat Hurmuz al-Malik. Mais c'est Sījān qui s'imposa comme nouvelle capitale, dominée par une citadelle naturelle qu'on fortifia solidement : Tīmūr sera le premier capable de s'en emparer en 1394. Au 10 s. Abū 'Alī Muhammad b. Ilyās, général rebelle aux sāmānides se retrancha dans la base qu'il édifia à Bardsīr et qu'il défendit par un système de fortifications commandant les routes alentour: Ghubayrā défendait l'accès vers le sud. La ville de Kirmān, à proximité de Bardsīr, s'imposa comme capitale régionale au $12^{\mathrm{e}} \mathrm{s}$.

2 Le site de Ghubayrāa à $70 \mathrm{~km}$ au sud de Kirmān, se trouve au confluent de la rivière pérenne Chārī et du wadi qui a donné son nom au site. Si peu de vestiges anciens ont pu être repérés dans les environs, des toponymes tels que Bahrāmjird ou Deh Gabrī évoquent le passé pré-islamique de l'endroit. A $8 \mathrm{~km}$ à l'ouest de Ghubayrā se trouve le village de Nigār qui garde d'un passé plus prospère un minaret dont la belle inscription de briques émaillées de l'époque seljūqide est l'un des plus anciens exemples attestant de cette technique en Iran.

3 Malgré son caractère spectaculaire, le site échappa à l'attention de l'explorateur passionné que fut Sykes et "Ghubera " apparut pour la première fois sur le relevé topographique effectué lors de sa reconnaissance du sud est iranien par A. Stein, mais 
comme village et non comme site archéologique. Ce n'est qu'à l'aube des années 70 qu'il fut reconnu comme tel par D. W. Chase, G. Fehérvári et J. R. Cadwell et c'est peu après que le centre iranien de recherche archéologique et la SOAS entreprirent de le fouiller.

4 Les vestiges étaient éparpillés sur une étendue de près de 50 ha. Au nord, près du confluent des deux cours d'eaux, des vestiges proto-historiques ont été mis au jour tandis que sur les éminences du sud se trouvaient la citadelle avec à l'ouest et au sud, deux mausolées de plan octogonal, probablement pas antérieurs à l'époque tīmūride, au nord, un enclos (le "Kishmish khāna ») et à l'ouest une autre ruine mineure. Le caractère morcelé du site a amené les auteurs de la présente publication à présenter les résultats de cette fouille campagne par campagne (il y en eut quatre, de 71 à 76) et à l'intérieur de chacune d'elles, à en présenter chaque opération, une trentaine, documentée par les notes de fouille. Il en résulte que le lecteur doit lui même opérer le regroupements des informations, secteur par secteur.

5 Le projet initial de l'équipe fut de fouiller la citadelle. Mais sa muraille étant par endroits disparue, à d'autres très érodée, il fut impossible d'en reconstituer le tracé. Une résidence ou palais fut mis au jour dans la partie sud de l'enceinte. Sur un soubassement de pierres taillées, ses murs en briques crues recouverts de plâtre formaient trois longues pièces parallèles sans doute bordées par une cour à portique dont deux colonnes fragmentaires étaient encore en place. Ce bâtiment, à l'origine pourvu d'un étage, pourrait appartenir à l'époque muzaffaride.

6 Dans le secteur de la citadelle furent aussi mis au jour une petite mosquée de conception rudimentaire et, une centaine de mètres au nord-ouest, un enclos rectangulaire de

18 x 38 m, sommairement bâti. Le nom qui le désigne localement, « Kishmish khāna », maison des raisins, éveilla la curiosité des fouilleurs qui ne purent néanmoins en découvrir la fonction. Seule la présence de carreaux de faïence gisant sur le sol suggère que certains murs en avaient sans doute été revêtus.

7 Si la fouille de la citadelle ne donna lieu qu'à des découvertes d'importance limitée, elle en livra cependant une, étonnante : un véritable labyrinthe de pièces souterraines et de corridors ramifiés, fouillé ou reconnu sur environ $800 \mathrm{~m}^{2}$. Ces installations souterraines étaient taillées dans les couches de grès alternant avec celles d'argile et de graviers constituant le "tāfk», formation géologique caractéristique de cet endroit. Les habitations souterraines, construites ou taillées, sont fréquentes dans les régions chaudes du sud de l'Iran mais l'importance de ce complexe de «bỉkam» est ici remarquable. A l'origine peut-être des catacombes préhistoriques selon A. Bivar (p. 71) elles ont à coup sûr servi de nécropole à l'époque sassanide comme en témoignent des fragments d'ossuaires de terre cuite et des bords de récipients avec inscriptions pahlavi. Plus tard, lieu de refuge, cachette ou seulement habitations pour supporter les mois torrides, ces «bīkam» ont durant des siècles été habitées, les objets d'usage courant s'y accumulant et s'y mélangeant d'une époque à l'autre, rendant toute référence stratigraphique impossible.

8 Le catalogue des objets découverts durant les quatre campagnes de fouille constitue l'essentiel de cette publication, en volume ( 382 p. sur 508) et en intérêt. Citons d'abord un trésor de drachmes sassanides allant de Xusraw I (531-579) à Ardashīr III (628-630). Parmi les autres monnaies qui ont pu être déchiffrées, une était byzantine et datait du $12^{\mathrm{e}}$, une douzaine datait des $13^{\mathrm{e}}-14^{\mathrm{e}} \mathrm{s}$. Mais parmi les objets métalliques la plus belle trouvaille est un bol de bronze intact de $13,5 \mathrm{~cm}$ de diamètre orné dans sa partie 
supérieure, à l'intérieur comme à l'extérieur, d'un bandeau portant une inscription propitiatoire en caractères nasHī terminée par un décor de rinceaux et, dans sa partie inférieure, de médaillons à rosaces et figures fantastiques. Un autre fragment de bol en métal, également était orné de poissons et de décors floraux. Trouvé dans la partie résidentielle de la citadelle, le premier de ces objets a été daté de l'époque seljūqide. Plusieurs autres objets de métal, dont plusieurs cuillères ou petites louches, n'ont pu être datées car provenant des pièces souterraines.

Le catalogue des céramiques donne un aperçu de l'histoire du site et des vicissitudes qu'il a subies, avec une prédominance des spécimens datant de la fin $d u 12^{\mathrm{e}} / 13^{\mathrm{e}} \mathrm{s}$. au $14^{\mathrm{e}}$, en particulier les récipients et éléments de revêtement à décor en relief sous glaçure monochrome. La présence plus discrète des pièces de l'époque sassanide et du début de l'Islam reflète sans doute une occupation moindre du site à cette époque, occupation qui a pu également être partiellement oblitérée par les suivantes. Les céramiques peintes à l'engobe sous glaçure transparente de la fin de l'époque sāmānide montrent la continuité du Kirmān avec la production khorassanienne et centraleasiatique.

La collection de verres trouvés sur ce site est remarquable, incluant trois pièces entières. Plusieurs fragments, à décor taillé ou moulé, attestent de la richesse de certains habitants de la ville. Plusieurs semblent appartenir à l'époque sassanide, mais en raison de la stabilité des techniques de fabrications du verre, et de l'absence de contexte stratigraphique, la plupart de ces objets n'ont pu être datés.

On regrettera l'organisation de ce catalogue dans lequel il est souvent long de retrouver la notice d'un objet à partir de son dessin et de sa photographie. Il faut cependant souligner la qualité de la présentation (typographie, illustration) et saluer la publication de la fouille de Ghubayrā, quand bien d'autres restent inédites.

INDEX

Thèmes : 5.1. Monde iranophone

\section{AUTEURS}

MONIK KERVRAN

CNRS - Paris 